\title{
Intraparenchymal supratentorial arachnoid cyst: a case report
}

\author{
Domagoj Dlaka ${ }^{1}$, Marina Raguž ${ }^{1 *}$ (D, Danko Muller², Dominik Romić ${ }^{2}$, Fadi Almahariq', Jelena Dlaka ${ }^{3}$, \\ Anđelo Kaštelančić ${ }^{1}$ and Darko Chudy ${ }^{1}$
}

\begin{abstract}
Background: Intracranial arachnoid cysts are collections of cerebrospinal fluid within the arachnoid membrane and subarachnoid space of the cisterns and major cerebral fissures that account for about $1 \%$ of all intracranial lesions. Expansion of the cyst and compression on surrounding structures may became symptomatic, which reflects its size and anatomic distribution.

Case presentation: Here, we present a very rare case of patient with supratentorial intraparenchymal arachnoid cyst placed in the left frontal lobe without any communication with the subarachnoid space and ventricle and presented with clinical symptoms. The patient underwent fenestration of the lesion and was clinically improved.

Conclusions: Although the etiology and the enlargement mechanism of arachnoid cysts remain unclear, both conservative and surgical treatments are optional. According to size, anatomical location, neuroimaging, and clinical symptoms, an arachnoid cyst should be included in the differential diagnosis of primary intracerebral cysts.
\end{abstract}

Keywords: Intraparenchymal lesion, Arachnoid cyst, Primary intracerebral cysts

\section{Background}

Intracranial arachnoid cysts are collections of cerebrospinal fluid (CSF) covered by arachnoid cells and collagen that account for about $1 \%$ of all intracranial lesions [1-4]. Arachnoid cysts occurs within the arachnoid membrane and in subarachnoid space of the major cerebral fissures and arachnoid cistern, most commonly within the Sylvian fissure, while other sites include the quadrigeminal plate, sellar and suprasellar region, quadrigeminal cerebellopontine angle, posterior infratentorial midline cisterns, and convexities [2-5]. Arachnoid cysts may result from either developmental defects or inflammatory or traumatic processes, hemorrhage, chemical irritation, or tumors that result with CSF sequestration [2, 6]. While cysts are usually encountered as incidental asymptomatic findings, some of them can expand, can compress surrounding structures, and therefore became symptomatic, mostly in early childhood [3].

\footnotetext{
* Correspondence: marinaraguz@gmail.com

${ }^{1}$ Department of Neurosurgery, University Hospital Dubrava, Avenija Gojka

Suska 6, HR-10000 Zagreb, Croatia

Full list of author information is available at the end of the article
}

Neurological signs and symptoms reflect the size and anatomic distribution of arachnoid cyst as well as their impact on CSF flow.

To our best of knowledge, there have been only few reported cases of supratentorial intraparenchymal arachnoid cyst $[1,4-8]$. We present a case of an arachnoid cyst arising in the left frontal lobe without any communication with the subarachnoid space and ventricle.

\section{Case presentation}

A 74-year-old female patient presented with a 1-month history of vertigo, gait problems, nausea, and intermittent headache. There were no other symptoms nor were there any neurological deficits at the time of admission. Initial computerized tomography (CT) and magnetic resonance imaging (MRI) revealed a well-defined nonenhancing mass in the left frontal lobe. T1-weighted images showed a large cystic intra-axial mass measuring $3.7 \times 3.8 \mathrm{~cm}$ containing partly homogenous low signal intensity with part of denser fluid; after the administration of intravenous contrast, the mass showed no enhancement (Fig. 1). On the T2-weighted images, the lesion had homogeneously high signal intensity. No surrounding brain edema was present (Fig. 2 a, Fig. 4 a, c). 


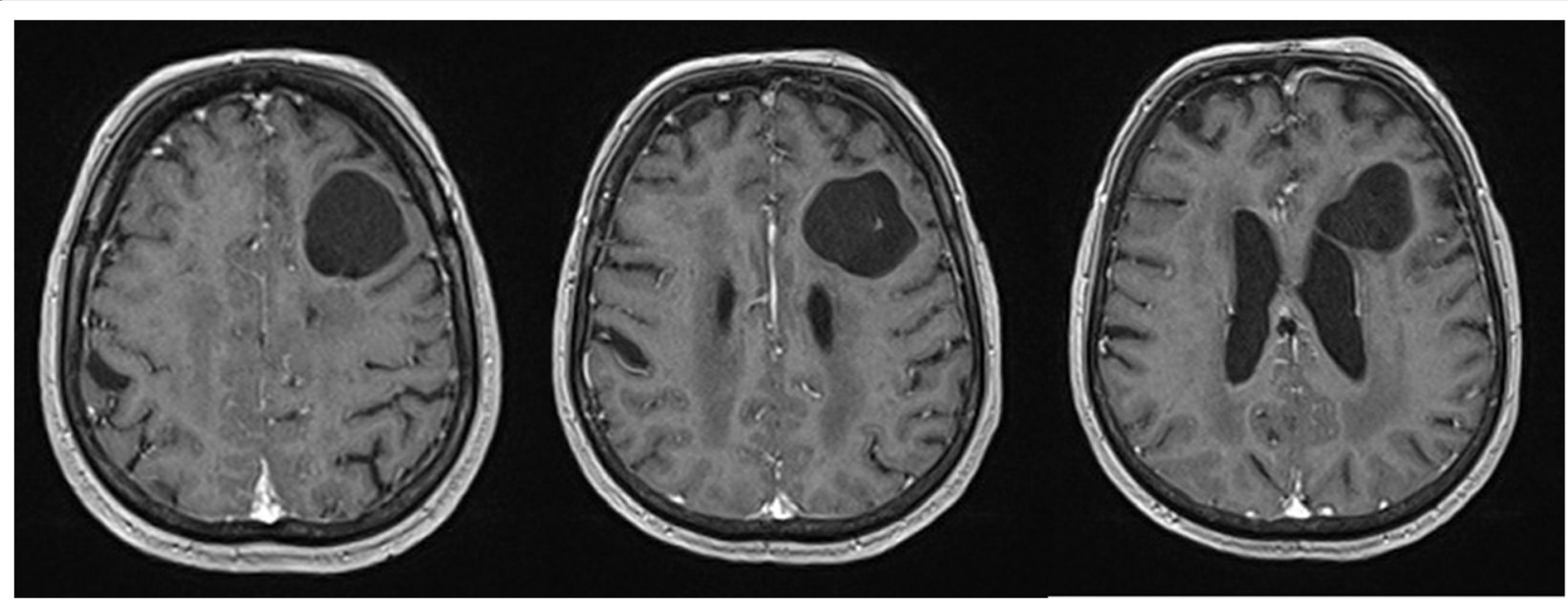

Fig. 1 Brain MRI showing left-sided large cystic intra-axial mass containing partly homogenous low signal intensity with part of denser fluid; after the administration of intravenous contrast, the mass showed no enhancement, T1-weighted image, transverse plain

Additionally, fluid attenuation inversion recovery (FLAIR) sequence showed that mass is comparable to the CSF and pointed towards differences in fluid density inside the cyst (Fig. 3a, c) (Additional file 1). The mass appeared to be situated entirely within the brain parenchyma, separated from both lateral ventricles and the subarachnoid space (Fig. 1 Fig. 2a; Fig. 3a, c; Fig. 4a, c).

Patient underwent a left-sided frontal craniotomy. During the surgery, the intraparenchymal cyst without communication with the arachnoid and lateral ventricle was identified. No visual difference in fluid density or color inside the cyst was observed. The cyst contained clear CSF-like fluid, which was evacuated. The cyst was opened widely; the cyst wall consisted exclusively out of white matter, and thin incomplete septa was observed within the cyst. The cyst was connected to the lateral ventricle by performing fenestration and resecting thin membrane separating it. Fluid sample, cyst wall sample, and septa within were collected for further examination. Surgery went without complications.

Pathohistological examination showed a delicate cystic structure lined by a flattened or cuboidal epithelium. On immunohistochemical staining, this

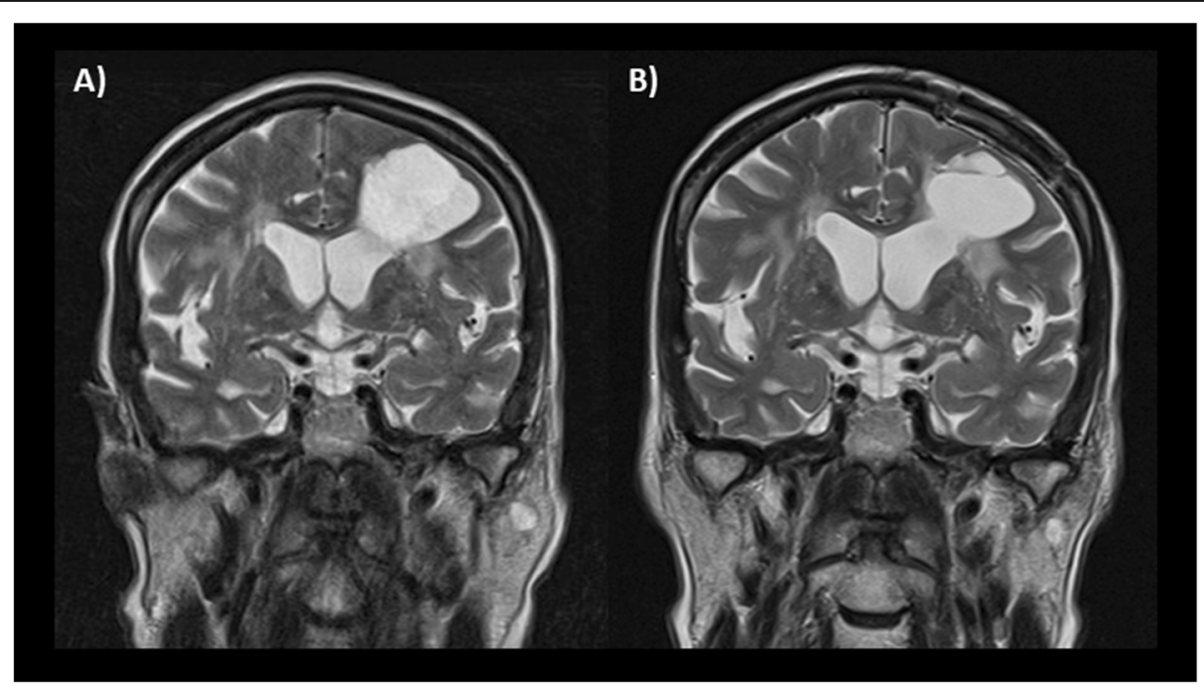

Fig. 2 Brain MRI showing left-sided well-defined mass, T2-weighted image: a preoperative scan, coronal plain, b postoperative scan, coronal plain 


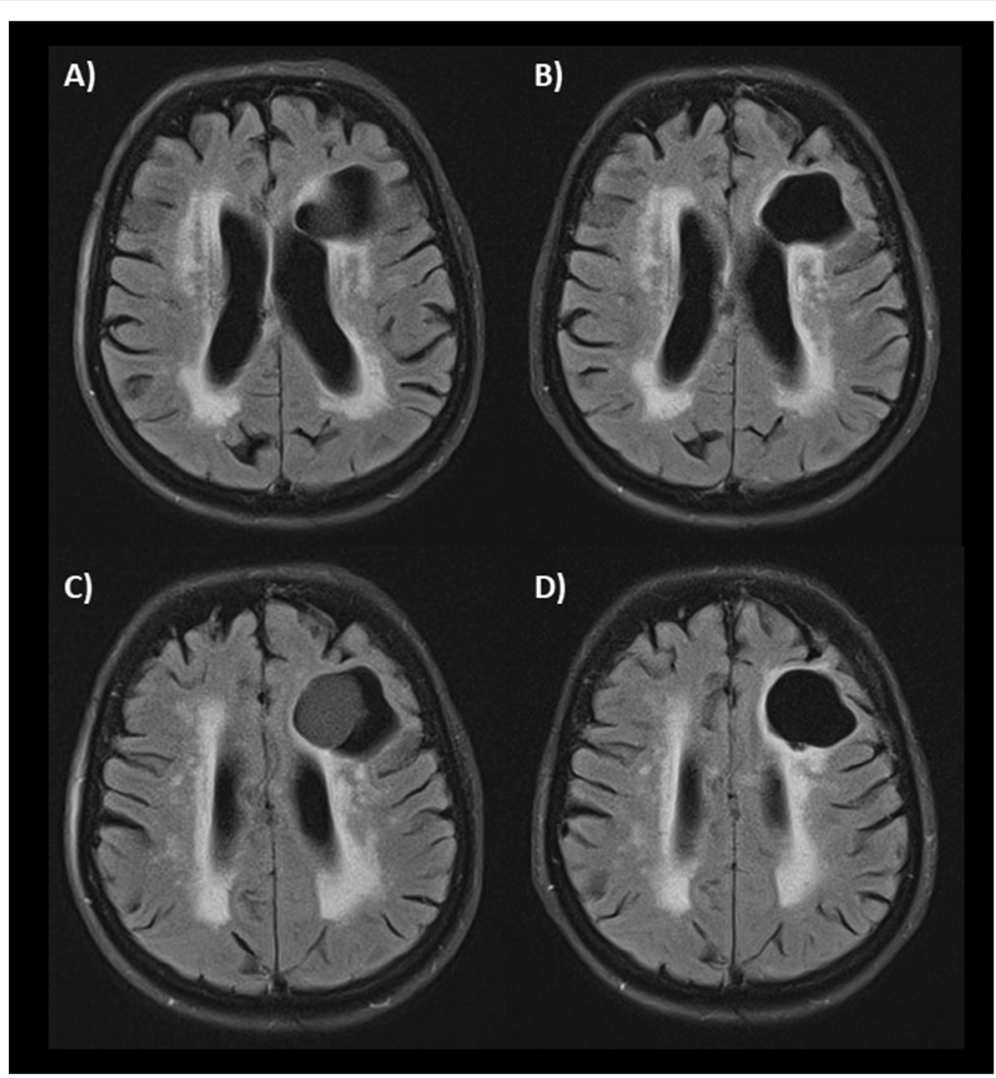

Fig. 3 Brain MRI showing left-sided well-defined mass, FLAIR sequence: a preoperative scan, transverse plain, $\mathbf{b}$ postoperative scan, transverse plain, $\mathbf{c}$ preoperative scan, transverse plain, $\mathbf{d}$ postoperative scan, transverse plain

epithelium showed positive reaction for epithelial membrane antigen (EMA) and negative for glial fibrillary acidic protein (GFAP) (Fig. 5). Analysis of the cystic fluid demonstrated a composition similar to that of normal CSF. Cytopathology of the fluid did not show the presence of any malignant cells or inflammatory cells. According to all mentioned, this cystic lesion was therefore diagnosed as an arachnoid cyst.

The patient did not show any neurological deficits in the postoperative period and was discharged 5 days after surgery. Three months afterwards, an MRI as well as a further neurosurgical control were performed. Initial neurological symptoms were improved, and MRI revealed a discrete reduction in cyst size without signs of complications (Fig. 2b; Fig. 3b, d; Fig. 4b, d).

\section{Conclusions}

The primary intracerebral cysts include ependymal, neurenteric, and epidermoid/dermoid cysts. Pathohistologically, arachnoid cyst is lined by flat or cuboidal cells overlying the fibrous connective tissue, unlike neurenteric cysts which are covered with simple to pseudostratified epithelium, epidermoid/dermoid cysts with keratinizing epithelium, or ependymal cysts in which case a basement membrane on the epithelial lining, which is present in arachnoid cysts, lacks $[1,4$, 9-11]. Immunohistochemically, the epithelium of an arachnoid cyst wall cells showed positive reaction for EMA and negative for GFAP, S-100, and carcinoembryonic antigen (CEA). Additionally, neurenteric cysts are usually positive for CEA, marker of the embryonic gastrointestinal tract, and negative for neuronal and ectodermal markers such as GFAP, S-100, neuronspecific enolase, and synaptophysin $[9,12-14]$. On the contrary, ependymal cysts show positive reaction for GFAP and negative for CEA [12, 13].

The differential diagnosis in-between primary intracranial cysts are very important for adequate treatment. Some symptomatic cysts, such as neurenteric and epidermoid/dermoid, should be properly surgically removed, including both cystic wall and content to avoid recurrences $[9,10]$. In our case, a parasitic echinococcosis cyst, cystic secondary lesion, or cystic 


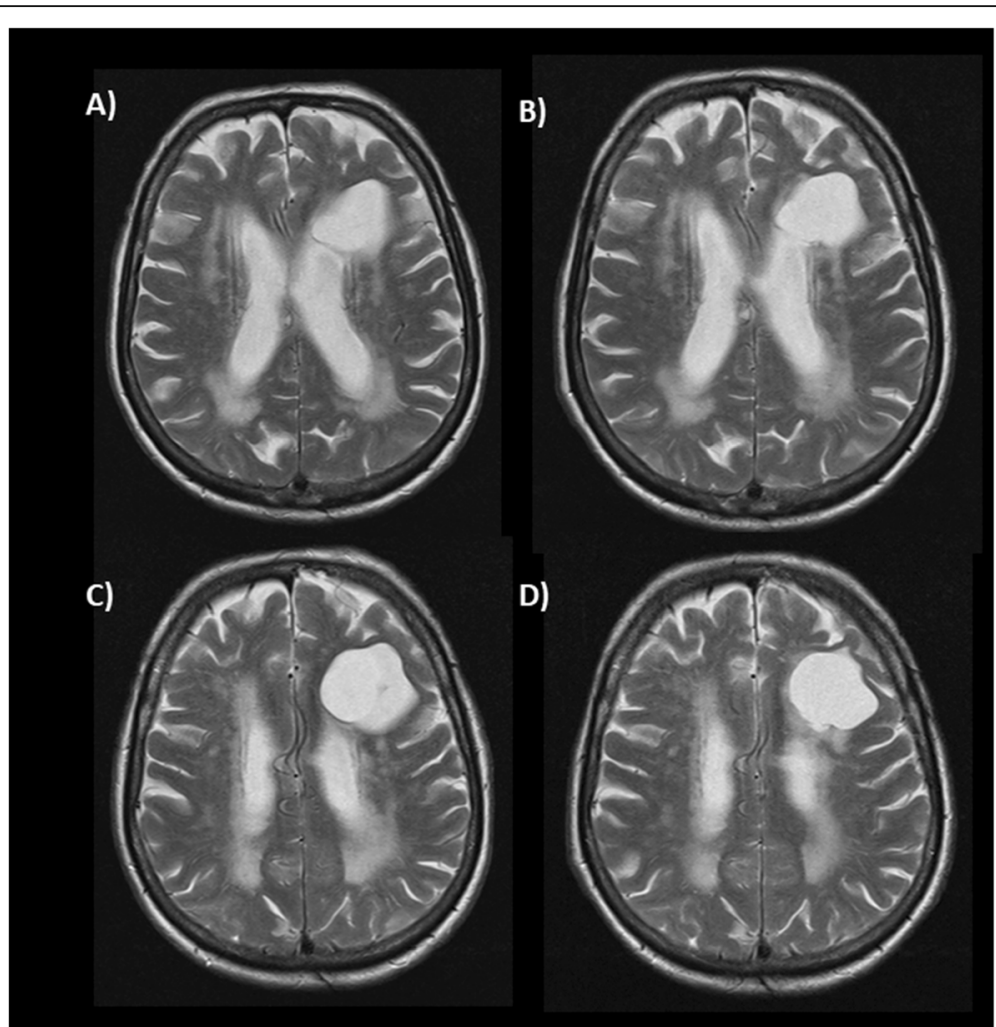

Fig. 4 Brain MRI showing left-sided well-defined mass, T2-weighted image: a preoperative scan, transverse plain, b postoperative scan, transverse plain, $\mathbf{c}$ preoperative scan, transverse plain, $\mathbf{d}$ postoperative scan, transverse plain

low-grade glioma was introduced in the differential diagnosis of lesions.

Several treatment options are currently optional for arachnoid cysts management, such as a craniotomy with resection of the cyst walls and content, stereotactic aspiration or fenestration of the cyst cavity, and neuroendoscopic fenestration or a shunting procedure $[4-6,15]$. If the cyst is located in a non-eloquent area and the resection of cyst from brain tissue is possible without damage, total excision of cyst, including both wall and contents, is the most appropriate treatment for symptomatic lesion [4].

Ongoing hypothesis for intraparenchymal cysts is that arachnoid cells of mesodermal origin can be present in neuroectodermal tissue as misplaced embryonic cellular remnants $[4,16]$. So far, in literature, there are only a few described cases of intracerebral cysts similar to arachnoid cyst $[1,4-8]$.

The etiology of arachnoid cysts yet remains unclear. Several theories have been proposed to explain the arachnoid cyst genesis such as corpus callosum agenesis, aberration in the development of the arachnoid, malformations of the cerebral venous systems, or CSF flow abnormalities [2-4]. Most of the arachnoid cysts remain constant through life; however, their enlargement cannot be explained with previously described theories, such as active CSF secretion from walls of the cysts, osmotic gradient between cystic content and CSF caused by a higher protein concentration in the cyst fluid, or cyst contents communicating with the subarachnoid space via ball-valve mechanisms that entrap CSF during Valsalva maneuvers [2, 17-19]. Previously mentioned mechanisms cannot explain neither the expansion of the presented cyst nor the missing communication between the cyst and subarachnoid space.

In the presented case, the establishment of diagnosis and treatment of the patient's symptoms were indications for surgery. Since no definitive diagnosis was made through neither clinical nor neuroradiological examinations, evacuation and resection of the cyst was performed with cyst fluid and wall sample collection. Although the symptoms are highly unspecific and cannot be completely linked to the existence of cyst or compressive effect of the cyst itself, the surgical treatment has provided improvement in the symptoms.

Since the majority of intracranial cyst remains in constant size and these are no neurological signs, conservative treatment and neuroradiological follow- 

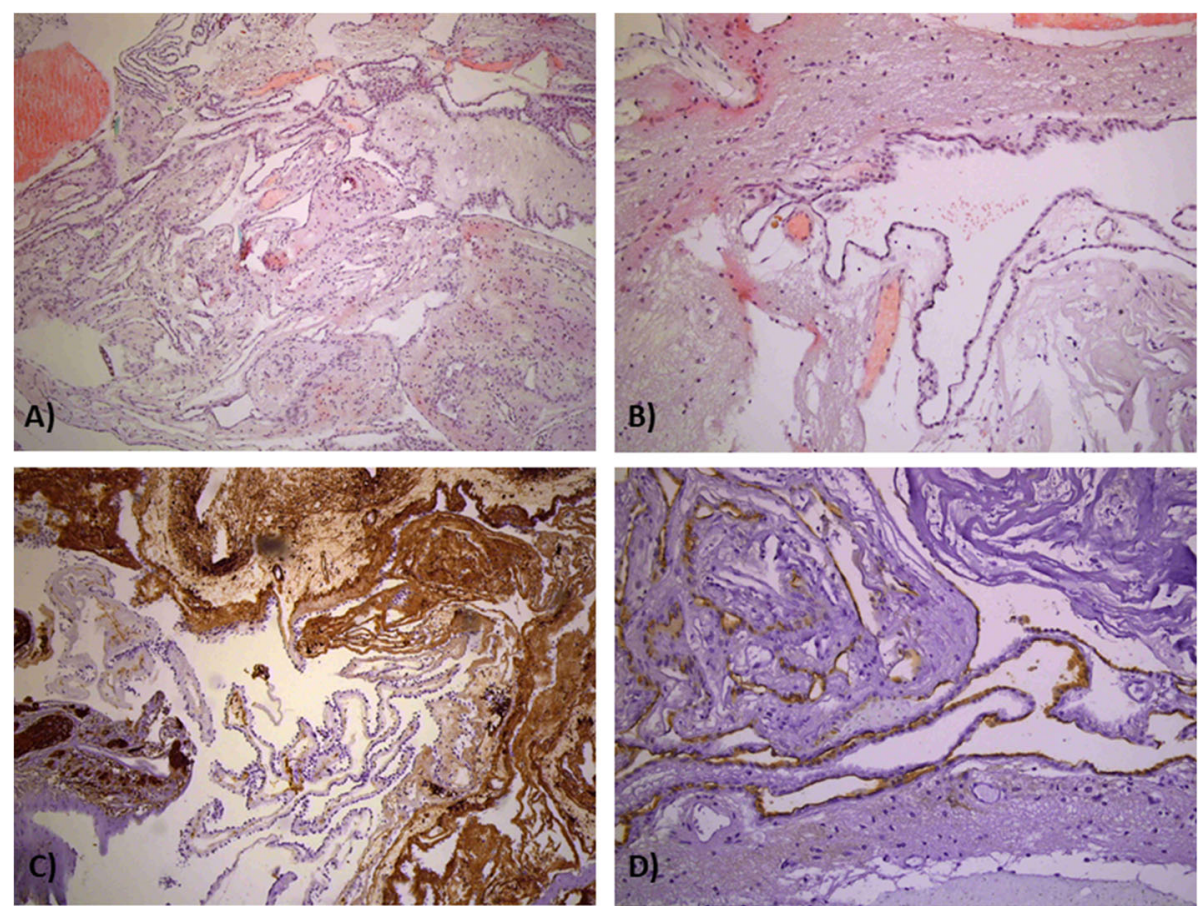

Fig. 5 Microphotography of a pathohistological section showing histological and immunohistochemical features of a delicate cystic structure lined by a flattened or cuboidal epithelium. Cystic wall stained with a hematoxylin and eosin, original magnification of $\times 100, \mathbf{b}$ hematoxylin and eosin, original magnification of $\times 200$. The epithelial cells showed no immunoreaction with glial fibrillary acidic protein (GFAP), c GFAP staining, original magnification of $\times 100$, and strong positive reaction for epithelial membrane antigen (EMA), $\mathbf{d}$ EMA staining, original magnification of $\times 200$

up have been proposed. So far, surgical treatment strategies for arachnoid cysts remain controversial. Despite unknown etiology and according to previously mentioned reasons, an arachnoid cyst should be included in the differential diagnosis of primary intracerebral cysts.

\section{Supplementary information}

Supplementary information accompanies this paper at https://doi.org/10. 1186/s41984-019-0053-8

Additional file 1: Figure S1. Comparison between preoperative T2 weighted images (A), transverse (first column) and coronal plain (second column) and postoperative T2 weighted images (B) transverse (first column) and coronal plain (second column). Discrete reduction of cyst size is observed.

\section{Abbreviations}

CEA: Carcinoembryonic antigen; CSF: Cerebrospinal fluid; CT: Computerized tomography; EMA: Epithelial membrane antigen; FLAIR: Fluid attenuation inversion recovery; GFAP: Glial fibrillary acidic protein; MRI: Magnetic resonance imaging

\section{Acknowledgements}

Not applicable.

\section{Authors' contributions}

DD and MR contributed to the study concept and design and wrote the first draft of the manuscript. DR, FA, and JD obtained the collection of clinical data presented. AK obtained all the MRI data and designed the figures. DM obtained the pathohistological data and designed the histological figure. DC contributed to the study concept and design and revised the manuscript. All authors approved the final version of the manuscript as submitted.

\section{Funding}

Not applicable.

\section{Availability of data and materials}

Data sharing is not applicable to this article as no datasets were generated or analyzed during the current study.

\section{Ethics approval and consent to participate}

Compliance with Ethical Standards, ethics approval, and consent were obtained from the Institutional Review Board of the University Hospital Dubrava, Zagreb, Croatia.

\section{Consent for publication}

The patient has given an informed consent for participation in this paper.

\section{Competing interests}

The authors declare that they have no competing interests.

\section{Author details}

'Department of Neurosurgery, University Hospital Dubrava, Avenija Gojka Suska 6, HR-10000 Zagreb, Croatia. ${ }^{2}$ Department of Pathology and Cytology, University Hospital Dubrava, Zagreb, Croatia. ${ }^{3}$ Intensive Care Unit, University Hospital Dubrava, Zagreb, Croatia. 
Received: 5 May 2019 Accepted: 16 September 2019

Published online: 09 October 2019

References

1. Iglesias JR, Sanjuanbenito L, Martinez-Cubells J, Lousa M, Redondo C. Intracerebral arachnoidal cyst containing choroid plexus: case report. Acta Neurochir. 1981;58(3-4):229-34

2. Cincu R, Agrawal A, Eiras J. Intracranial arachnoid cysts: current concepts and treatment alternatives. Clin Neurol Neurosurg. 2007;109(10):837-43.

3. Pradilla G, Jallo $G$. Arachnoid cysts: case series and review of the literature. Neurosurg Focus. 2007;22(2):E7.

4. Park KJ, Kang SH, Chae YS, Chung YG. Supratentorial arachnoid cyst located in the brain parenchyma: case report. Neurosurgery. 2011;68(1):E258-62

5. Gandy SE, Heier LA. Clinical and magnetic resonance features of primary intracranial arachnoid cysts. Ann Neurol. 1987;21(4):342-8.

6. Choi JU, Kim DS. Pathogenesis of arachnoid cyst: congenital or traumatic. Pediatr Neurosurg. 1998;29(5):260-6.

7. El Ghandour NM. Endoscopic treatment of intraparenchymal arachnoid cysts in children. J Neurosurg Pediatr. 2014;14(5):501-7.

8. Mino M, Fujimura M, Tominaga T. Neuro-endoscopic management of intraparenchymal arachnoid cyst in adults: three case reports. No Shinkei Geka. 2019:47(4):461-7.

9. Miyagi A, Katayama Y. Neurenteric cyst arising in the high convexity parietal lesion: case report. Neurosurgery. 2007;60(1):E203-4.

10. Eynon-Lewis NJ, Kitchen N, Scaravilli F, Brookes GB. Neurenteric cyst of the cerebellopontine angle: case report. Neurosurgery. 1998:42(3):655-8.

11. Burger PC, Scheithauer BW. Tumors of the central nervous system. Washington: US Armed Forces Institute of Pathology, Universities Associated for Research and Education in Pathology; 1994

12. Inoue T, Matsushima T, Fukui M, Iwaki T, Takeshita I, Kuromatsu C. Immunohistochemical study of intracranial cysts. Neurosurgery. 1988;23(5):576-81.

13. Graziani N, Dufour H, Figarella-Branger D, Donnet A, Bouillot P, Grisoli F. Do the suprasellar neurenteric cyst, the Rathke cleft cyst and the colloid cyst constitute a same entity? Acta Neurochir. 1995;133(3-4):174-80.

14. Kachur E, Ang LC, Megyesi JF. Intraparenchymal supratentorial neurenteric cyst. Can J Neurol Sci. 2004;31(3):412-6.

15. Boutarbouch $M$, El Ouahabi A, Rifi L, Arkha Y, Derraz S, El Khamlichi A. Management of intracranial arachnoid cysts: institutional experience with initial 32 cases and review of the literature. Clin Neurol Neurosurg. 2008;110(1):1-7.

16. Hyndman OR, Gerber WF. Spinal extradural cysts, congenital and acquired: report of cases. J Neurosurg. 1946;3(6):474-86.

17. Gosalakkal JA. Intracranial arachnoid cysts in children: a review of pathogenesis, clinical features, and management. Pediatr Neurol. 2002;26(2):93-8.

18. Schachenmatr W, Friede RL. Fine structure of arachnoid cysts. J Neuropathol Exp Neurol. 1979:38(4):434-46.

19. Santamarta D, Aguas J, Ferrer E. The natural history of arachnoid cysts: endoscopic and cine-mode MRI evidence of a slit-valve mechanism. Invasive Neurosurg. 1995;38(4):133-7.

\section{Publisher's Note}

Springer Nature remains neutral with regard to jurisdictional claims in published maps and institutional affiliations.

\section{Submit your manuscript to a SpringerOpen ${ }^{\circ}$ journal and benefit from:}

- Convenient online submission

- Rigorous peer review

Open access: articles freely available online

- High visibility within the field

- Retaining the copyright to your article

Submit your next manuscript at $\boldsymbol{\nabla}$ springeropen.com 